\title{
Extragalactic Background Light expected from photon-photon absorption on spectra of distant Active Galactic Nuclei
}

\author{
V.G. Sinitsyna ${ }^{1, a}$ and V.Y. Sinitsyna ${ }^{1}$ \\ ${ }^{1}$ P. N. Lebedev Physical Institute, Russian Academy of Science
}

\begin{abstract}
Extragalactic background radiation blocks the propagation of $\mathrm{TeV}$ gamma-ray over large distances by producing $e^{+} e^{-}$pairs. As a result, primary spectrum of gamma-source is changed, depending on spectrum of background light. So, hard spectra of Active Galactic Nuclei with high red shifts allow the determination of a EBL spectrum. The redshifts of SHALON TeV gamma-ray sources range from 0.018 to 1.375 those spectra are resolved at the energies from $800 \mathrm{GeV}$ to $30 \mathrm{TeV}$. Spectral energy distribution of EBL constrained from observations of Mkn421 ( $\mathrm{z}=0.031$ ), Mkn501 ( $\mathrm{z}=0.034)$, Mkn180 ( $\mathrm{z}=0.046)$, OJ287 ( $\mathrm{z}=0.306), 3 \mathrm{c} 454.3$ $(\mathrm{z}=0.859)$ and $1739+522(\mathrm{z}=1.375)$ together with models and measurements are presented.
\end{abstract}

\section{Introduction}

The cosmological processes, connecting the physics of matter in Active Galactic Nuclei will be observed in the energy spectrum of electromagnetic radiation. The understanding of mechanisms in active galactic nuclei requires the detection of a large sample of very high energy gamma-ray objects at varying redshifts. The detection of the very high energy $\gamma$-ray sources at the redshifts $\mathrm{z}=0.0179$ to $\mathrm{z}=1.375$ with SHALON telescope gives an opportunity to constrain extragalactic background light (EBL) density, based on modification to gamma-ray spectra and thus it will help to reconstruct the the cosmological history of the EBL.

\section{SHALON telescope}

The $\gamma$-astronomical researches are carrying out with SHALON [1-9] mirror Cherenkov telescope at the TienShan high-mountain observatory (fig. 1). The SHALON mirror telescopic system consists of composed mirror with area of $11.2 \mathrm{~m}^{2}$. It is equipped with 144 photomultipliers receiver with the pixel of $0.6^{\circ}$ and the angular resolution of the experimental method of $<0.1^{\circ}$. It is essential that our telescope has a large matrix with full angle $>8^{\circ}$ that allows us to perform observations of the supposed astronomical source (ON data) and background from extensive air showers (EAS) induced by cosmic ray (OFF data) simultaneously. Thus, the OFF data are collecting for exactly the same atmospheric thickness, transparency and other experimental conditions as the ON data.

An additional selection of electron-photon showers among the net cosmic rays EAS becomes possible through

\footnotetext{
a e-mail: sinits@sci.lebedev.ru
}

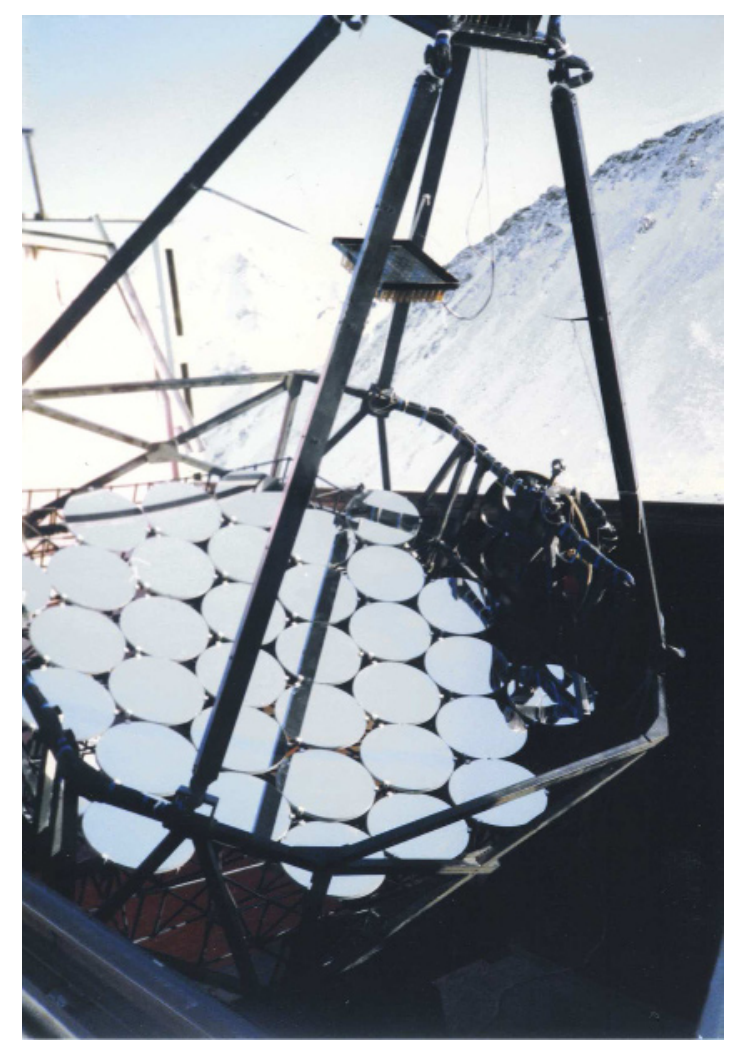

Figure 1. SHALON telescope.

an analysis of a light image which, in general, emerging as an elliptic spot in light receiver matrix. The selection of gamma-initiated showers from the background of proton showers is performed by applying the following criteria: 
Table 1. The catalogue of metagalactic $\gamma$-ray sources observed by SHALON.

\begin{tabular}{cccccc}
\hline Sources & Observable flux $^{a}$ & $k_{\gamma}{ }^{b}$ & Distance $^{c}$ & $\mathrm{z}$ & Type \\
\hline NGC 1275 & $(0.78 \pm 0.05)$ & $-2.24 \pm 0.09$ & 71 & 0.018 & Seyfert \\
SN2006 gy & $(3.71 \pm 0.65)$ & $-3.13 \pm 0.27$ & 83 & 0.019 & SN \\
Mkn 421 & $(0.63 \pm 0.05)$ & $-1.87 \pm 0.11$ & 124 & 0.031 & BL Lac \\
Mkn 501 & $(0.86 \pm 0.06)$ & $-1.87 \pm 0.13$ & 135 & 0.034 & BL Lac \\
Mkn 180 & $(0.65 \pm 0.09)$ & $-2.16 \pm 0.15$ & 182 & 0.046 & BL Lac \\
3c382 & $(0.95 \pm 0.20)$ & $-1.08 \pm 0.11$ & 247 & 0.0578 & BLRG \\
OJ 287 & $(0.26 \pm 0.07)$ & $-1.43 \pm 0.18$ & 1070 & 0.306 & BL Lac \\
3c454.3 & $(0.43 \pm 0.07)$ & $-0.85 \pm 0.07$ & 5489 & 0.859 & FSRQ \\
1739+522 & $(0.49 \pm 0.05)$ & $-0.93 \pm 0.09$ & 9913 & 1.375 & FSRQ \\
\hline \multicolumn{5}{c}{ Integral flux at energy > 800 GeV in units of $10^{-12} \mathrm{~cm}^{-2} \mathrm{~s}^{-1}$} & \\
\hline \multicolumn{5}{c}{${ }^{b}$ Distance in Mpc } \\
\end{tabular}

- $\alpha<20^{\circ}$;

- length/width > 1.6;

- the ratio INT0 of Cherenkov light intensity in pixel with maximum pulse amplitude to the light intensity in the eight surrounding pixels exceeds $>0.6$;

- the ratio INT 1 of Cherenkov light intensity in pixel with maximum pulse amplitude to the light intensity in the in all the pixels except for the nine in the center of the matrix is exceeds $>0.8$;

- distance is less than 3.5 pixels.

Our analysis of the distributions of listed shower image parameters suggests that the background is rejected with $99.92 \%$ efficiency (see Refs. [2, 3, 5, 7-9]), whereas the amount of background showers to the selected gammashowers does not exceed of $10 \%$.

The $\gamma$-astronomical researches are carrying out with SHALON [1] mirror Cherenkov telescope since 1992. During the period 1992 - 2012 SHALON has been used for observations of extragalactic sources of different type. Among them are known BLLac-type sources Mkn 421, Mkn 501, Mkn 180, OJ 287; Seyfert Galaxy NGC 1275; Broad Line Radio Galaxy 3c 382; and Flat Spectrum Radio Qusars: $3 c$ 454.3; 1739+522 (4c+51.37). [2-8]. Our method of the data processing is described in $[1-4,9]$. Some representative results on fluxes, spectra are shown in Table 1 and also in [2-8] and Figures in these proceedings.

\section{OJ 287}

OJ 287 ( $\mathrm{z}=0.306$ [11]) is an low-frequency peaked BL Lac objects. It is one of the most studied blazars which spectrum has been well measured through radio [12-14] to Xray bands [15-18] for radio studies and for optical. The most outstanding characteristic of OJ 287 is its 12 year period, which is discovered in optical range [16] and has also been confirmed in the X-ray band. OJ 287 is supposed to be a binary black hole system in which a secondary black

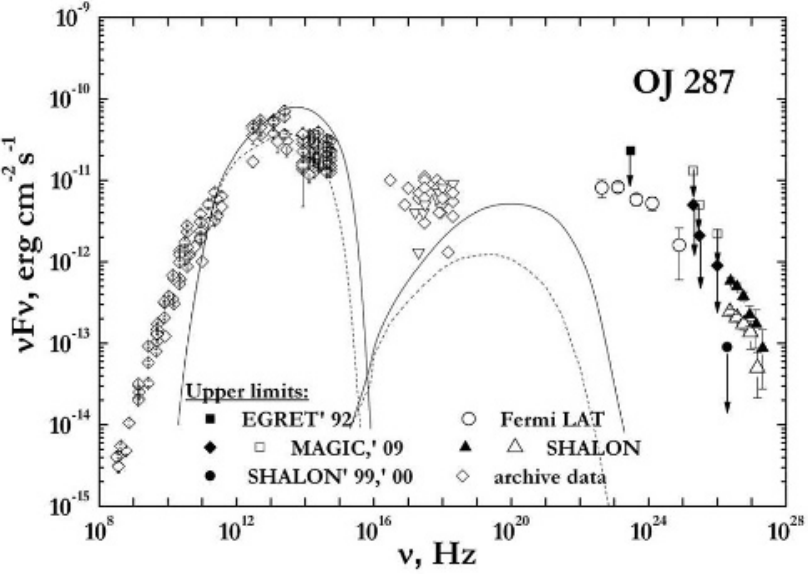

Figure 2. The spectral energy distribution of OJ 287 (see text).

hole passes the accretion disk of the primary black hole and produces two impact flashes per period. The spectral energy distributions of blazars consist of two broad peaks. The first, lower frequency peak is due to the synchrotron emissions of relativistic electrons in the jet. It is supposed, that the second, higher frequency peak is to be due to the Inverse Compton emissions of the same electrons (also refered as "synchrotron self-Compton model [19]). OJ 287 has been detected with $\mathrm{GeV}$ emissions by EGRET [20] and Fermi LAT[21]. It has also been proposed to be a TeV source.

OJ 287 was observed by SHALON in 1999, 2000, 2008, 2009 and 2010, for a total of 47.3 hours, at zenith angles ranging from $22^{\circ}$ to $34^{\circ}$. The observations of 1999 and 2000 years does not reveal a $\gamma$-ray flux from the position of OJ 287, but only an upper limit of < $1.1 \times 10^{-13} \mathrm{~cm}^{-2} \mathrm{~s}^{-1}$ (fig. 2). In observations of 2008, 2009 and 2010 (31.2 hours in total) the weak $\gamma$-ray flux was detected. An excess corresponding to a $6.9 \sigma$ [10] was determined. The measured integral energy spectrum of OJ 287 can be well described by a power law with the index $-1.43 \pm 0.18$ (fig. 3). The observed integral flux above 

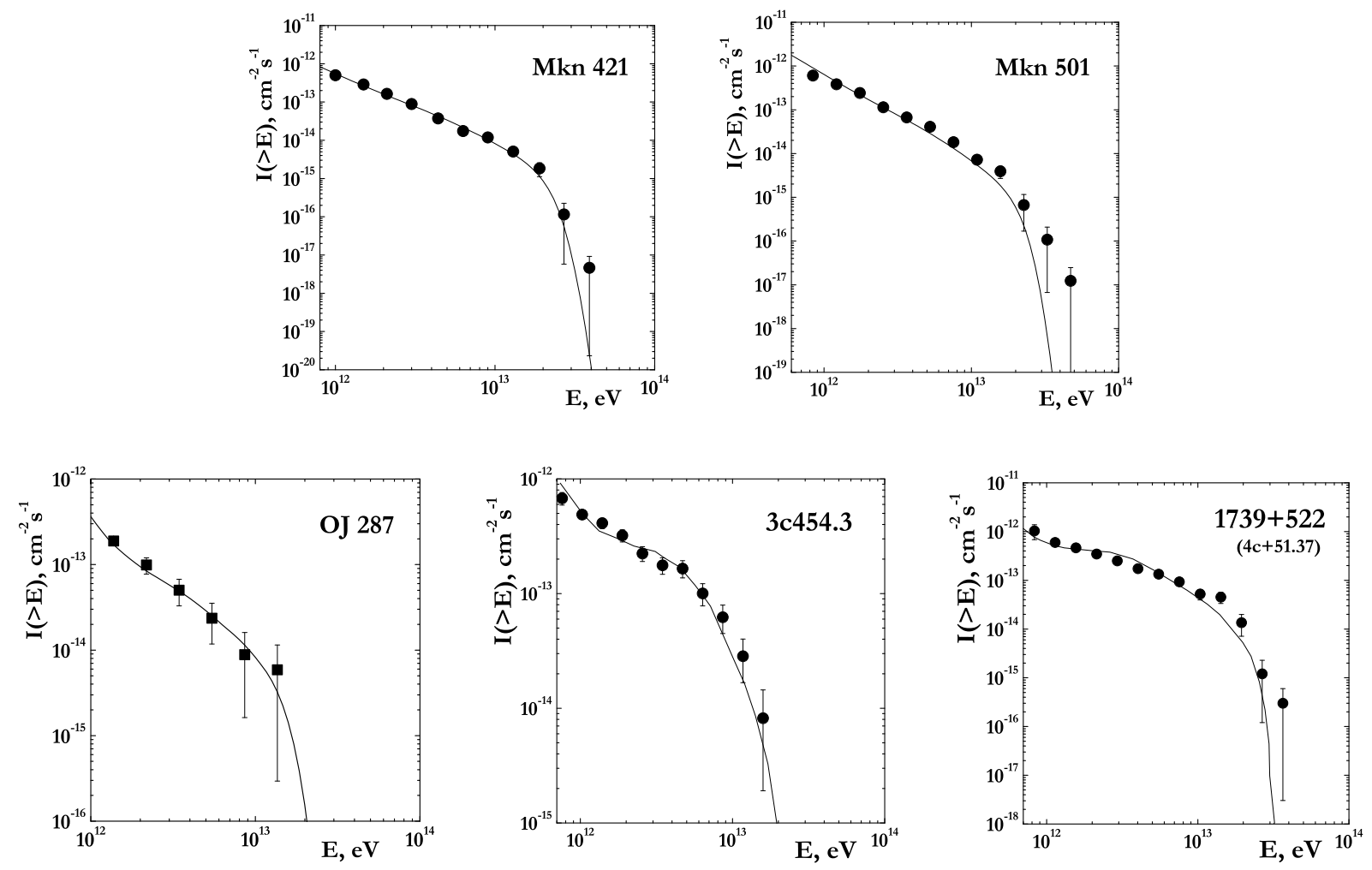

Figure 3. The integral energy spectra for Mkn 421, Mkn 501, OJ287, 3c454.4 and 1739+522 measured by SHALON (black points) together with spectra attenuated by EBL (lines, see text).

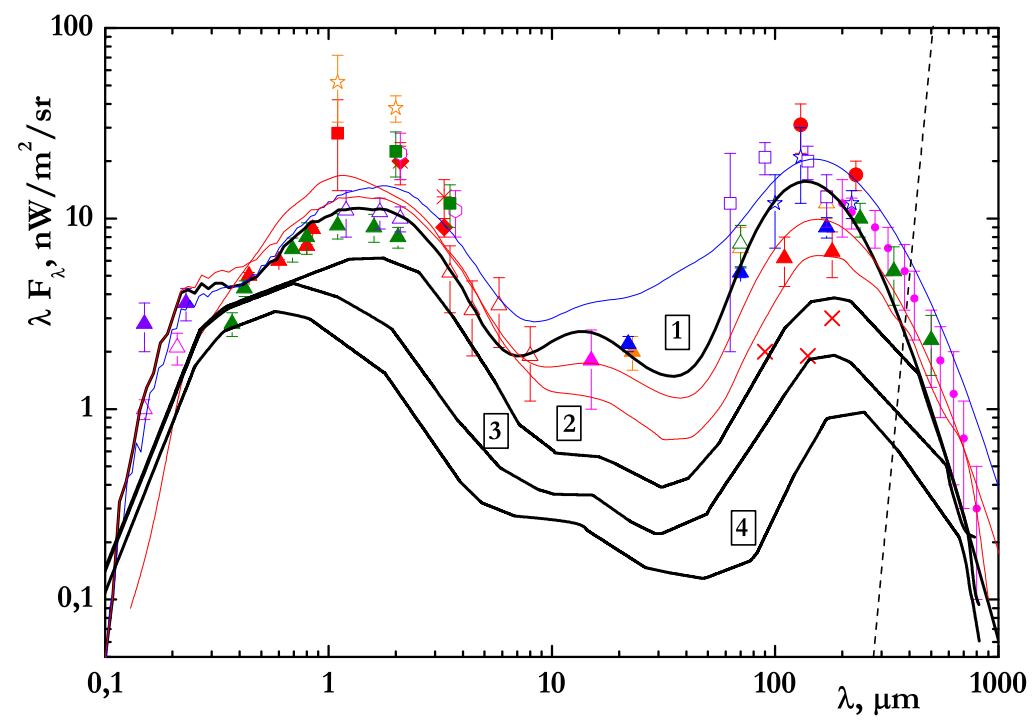

Figure 4. Spectral energy distribution of EBL: measurements [30] and models [31-34] and EBL shape constrained from observations of the extragalactic sources by SHALON:

1 - Mkn 421 (z=0.031), Mkn 501 ( $\mathrm{z}=0.034) ; 2$ - OJ287 ( $\mathrm{z}=0.306) ; 3$ - 3c454.3(z=0.859); 4 - 1739+522 ( $\mathrm{z}=1.375)$.

$800 \mathrm{GeV}$ is $(0.26 \pm 0.07) \times 10^{-12} \mathrm{~cm}^{-2} \mathrm{~s}^{-1}$. An image of $\gamma$ ray emission from OJ 287 by SHALON telescope is also obtained.
The flux increase over the detected average flux was found at 14,15 November and 4, 5 December 2010 with value of $(0.63 \pm 0.15) \times 10^{-12} \mathrm{~cm}^{-2} \mathrm{~s}^{-1}$ (statistical significance of $6.2 \sigma[10])$ with the softer energy spectrum with a 
power law with the index $-1.96 \pm 0.16$. Figure 2 presents spectral energy distribution [22] of the $\gamma$-ray emission from OJ 287 by SHALON in comparison with other experiment data MAGIC [22], EGRET [20], and with theoretical predictions from [21] and also [22]. The open triangles at $\mathrm{TeV}$ energies on Fig. 2 are SHALON spectrum of OJ287; an upper limit at $>0.8 \mathrm{TeV}$ corresponds to SHALON observations in 1999, 2000. The black triangles present the $\gamma$-ray spectrum at the increased flux period of 2010. OJ 287 is the weakest extragalactic source observed by SHALON.

\section{$43 c 454.3$}

In 1998 year a new metagalactic source $3 \mathrm{c} 454.3(\mathrm{z}=0.859)$ had been detected by SHALON at TeV energies [2-7]. The integral gamma-ray flux above $0.8 \mathrm{TeV}$ was estimated as $(0.43 \pm 0.07) \times 10^{-12} \mathrm{~cm}^{-2} \mathrm{~s}^{-1}$ (Table 1, Fig. 3, bottom line). Within the range $0.8-9 \mathrm{TeV}$, the integral energy spectrum is well described by the single power law $I\left(>E_{\gamma}\right) \propto E_{\gamma}^{-0.85 \pm 0.07}$ (fig. 3). Also, the spectrum by SHALON within the energy range $0.8-17 \mathrm{TeV}$ can fitted with a power-law spectrum with exponential cut-off.

The measured flux value is consistent with the upper limit $0.84 \times 10^{-11} \mathrm{~cm}^{-2} \mathrm{~s}^{-1}$ obtained by Whipple telescope at energy more than $0.5 \mathrm{TeV}$ [23-26]. Taking into account that the spectrum from $3 \mathrm{c} 454.3$ measured by EGRET in the energy range from $\sim 30 \mathrm{MeV}$ to $50 \mathrm{GeV}$ can be approximated as $E^{-1.2}$ [27].

Recently, 3c454.3 has detected with Fermi LAT at energies $200 \mathrm{MeV}-300 \mathrm{GeV}$ [28]. The spectrum by Fermi is fitted with a broken power-law with photon indices $\Gamma_{\text {low }}=2.27 \pm 0.3, \Gamma_{\text {high }}=3.5 \pm 0.05$ with an average flux of $\sim 3 \times 10^{-6}$ photons $\mathrm{cm}^{-2} \mathrm{~s}^{-1}$, for energies $>100 \mathrm{MeV}$.

\section{$51739+522$}

One more remote metagalactic gamma - source was detected by SHALON in 1999 and is being intensively studied since then [2-7]. This object was identified with the active galactic nucleus $1739+522$. This the most distant object (with redshift $\mathrm{z}=1.375$ ) is also the most powerful: its integral gamma-ray flux is found to be $(0.49 \pm 0.05) \times$ $10^{-12} \mathrm{~cm}^{-2} \mathrm{~s}^{-1}$ at energies of $>0.8 \mathrm{TeV}$. Within the range $0.8-7 \mathrm{TeV}$, the integral energy spectrum is well described by the single power law $I\left(>E_{\gamma}\right) \propto E_{\gamma}^{-0.93 \pm 0.09}$ (fig. 3, bottom line). Also, within the energy range $0.8-35 \mathrm{TeV}$ the average integral spectrum can fitted with a power-law spectrum with exponential cut-off.

The average gamma-flux measured by EGRET telescope of Compton Observatory (CGRO) in the range $\sim 30$ $\mathrm{MeV}$ to $50 \mathrm{GeV}$ is about $2 \times 10^{-8} \mathrm{~cm}^{-2} \mathrm{~s}^{-1}$ with integral spectrum index about -1.2 [27]. The high energy $\gamma$-ray emission from $1739+522$ was detected with Fermi LAT [29] in the range $100 \mathrm{MeV}-100 \mathrm{GeV}$ with an average flux of $F(>1 \mathrm{GeV})=(2.5 \pm 0.2) \times 10^{-9} \mathrm{~cm}^{-2} \mathrm{~s}^{-1}$ and power-law with photon indices $\Gamma=2.5 \pm 0.04$ [29]

According to our analysis, the energy spectra of distant quasars $3 \mathrm{c} 454.3(\mathrm{z}=0.859)$ and $1739+522(\mathrm{z}=1.375)$ :
$F_{3 c 454.3}\left(>E_{\gamma}\right) \propto E_{\gamma}^{-0.85 \pm 0.07}$ and $F_{1739+522}\left(>E_{\gamma}\right) \propto$ $E_{\gamma}^{-0.93 \pm 0.05}$ differ from those of the known blazars Mkn $421(\mathrm{z}=0.031)$ and $\operatorname{Mkn} 501(\mathrm{z}=0.034): F_{M k n} 421\left(>E_{\gamma}\right) \propto$ $E_{\gamma}^{-1.87 \pm 0.11}$ and $F_{M k n 501}\left(>E_{\gamma}\right) \propto E_{\gamma}^{-1.85 \pm 0.11}$. Hence, the average energy spectrum of the nearest metagalactic sources differs from spectra of remote objects $1739+522$ and $3 \mathrm{c} 454.3$ within the energy range $10^{12}-10^{13} \mathrm{eV}$. This observation of distant quasars does not contradict to unified energy spectrum $F\left(>E_{\gamma}\right) \propto E_{\gamma}^{-1.2 \pm 0.1}$.

\section{Extragalactic Background Light}

As the $\mathrm{TeV}$ gamma rays can be absorbed due to interaction of low-energy photons of Extragalactic Background Light (EBL), the observations of active galactic nuclei can also be used for the study background light from UV to far infrared and even cosmic microwave background. $\mathrm{TeV}$ gamma-rays, radiated by distant sources, interact with photons of background via $\gamma+\gamma \rightarrow e^{+} e^{-}$resonant process, then relativistic electrons can radiate gamma-ray with energies less than of primary gamma-quantum. As a result, primary spectrum of gamma-source is changed, depending on spectrum of background light. So, a hard spectra of AGNi with high red shifts of $0.03-1.8$ allow the determination an absorption by EBL and thus its spectrum.

The redshifts of SHALON very high energy gammaray sources range from $\mathrm{z}=0.018$ to $\mathrm{z}=1.375$. Among them bright enough AGNi of BLLac type Mkn421, Mkn 501 and FSRQ type 3c454.3, 1739+522 $(4 c+51.37)$ those spectra are resolved in the TeV energy band from $800 \mathrm{GeV}$ up to $\sim 20-50 \mathrm{TeV}$. The fit of a simple power law function to the observational data presented in Table 1. Also, the measured spectra can be fitted by a power law with an exponential cutoff: $F(>E) \propto E^{-\gamma} \times \exp \left(-E / E_{\text {cutoff }}\right)$ with hard power indices of about $\gamma \sim 1.55$ for Mkn 421 and Mkn 501 and $\gamma \sim 0.6$ for $3 \mathrm{c} 454.3$ and $1739+522$. The value of $E_{\text {cutoff } f}$ ranges from $11 \pm 2 \mathrm{TeV}$ for Mkn 421, Mkn 501 and to $7 \pm 2 \mathrm{TeV}$ for distant sources.

It has mentioned that the observed spectra are modified by $\gamma$-ray attenuation, i.e. $F_{\text {observed }}(E)=F_{\text {intrinsic }}(E) \times$ $\exp (-\tau(E, z))$ where $\tau(E, z)$ is optical depth for pair creation for a source at redshift $z$, and at an observed energy $E$. According to the definition of the optical opacity the medium influences on the primary source spectrum at $\tau \geq 1$, but for $\tau<1$ the medium is transparent, so the measuring of source spectrum in the both range of $\tau$ can give the intrinsic spectrum of the source to constrain the EBL density. The optical depth for sources at redshifts from 0.031 to 1.375 was calculated with assumption of EBL shapes shown in Fig. 4. We used the EBL shape from Bestfit model and Low-SFR model [33] (see Fig. 4 thick black line 1 corresponds to Low-SFR model) to calculate the attenuated spectrum of Mkn 421 and Mkn 501 in assumption of simple power low intrinsic spectrum of the source with spectrum index of $\gamma=1.5$, taken from the range of $\tau<1$. The result is shown at Fig. 3 with line; the black squares are observational data for Mkn 421 and Mkn 501. The shapes of EBL density constrained from the spectra of the high redshift sources OJ287 ( $\mathrm{z}=0.306), 3 \mathrm{c} 454.3(\mathrm{z}=0.859)$ 
and 1739+522 (1.375) are shown in Fig. 4 with curves 2, 3 and 4 , respectively. For these sources the slope of intrinsic spectrum is taken $\gamma=0.9-1.2$. The attenuated spectra for OJ287, 3c454.3 and 1739+522 are also presented at Fig. 3 (thin lines) together with observational data.

Observations of distant metagalactic sources have shown that the Universe is more transparent to very highenergy $\gamma$-rays than previously believed.

\section{References}

[1] S. I. Nikolsky and V. G. Sinitsyna, in Proc. Int. Workshop on VHE $\gamma$-ray Astronomy, Crimea, ed. A. A. Stepanian et al. 11 (1989)

[2] V. G. Sinitsyna et al., Int. J. Mod. Phys. A 29, 7023, 7026, 7029 (2005).

[3] V. G. Sinitsyna et al., Nucl. Phys. B (Proc. Suppl.) 196, 251, 442 (2009); ibid. 175-176, 463 (2008); ibid. 151, 108 (2006); ibid. 122, 247, 409 (2003); ibid. 97, 215 and 219 (2001); ibid. 75A, 352 (1999).

[4] S. I. Nikolsky and V. G. Sinitsyna, Phys. Atom. Nucl. 67, 1900 (2004)

[5] V. G. Sinitsyna, AIP (Conf. Proc.) 515, 205 and 293 (1999).

[6] V. G. Sinitsyna, in Proc. Toward a Major Atmospheric Cherenkov Detector-IV, ed. M. Cresti (Papergraf PD), 133 (1995); in Detector-V, ed. O. C. De Jager (Wesprint, Potcherfstroom) 136 and 190 (1997); in Detector-VII, ed. B. Degrange, G. Fontain 57, 105, 11 (2005).

[7] V. G. Sinitsyna,et al., Izv. Ross. Akad. Nauk Ser. Fiz. 71(7), 94 (2007); ibid. 69(3), 422 (2005); ibid. 66(11), 1654 and 1661 (2002); ibid. 63(3), 608 (1999); ibid. 61(3), 603 (1997).

[8] V. G. Sinitsyna, et al., in Proc. The Universe Viewed in Gamma-Rays, ed.R. Enomoto, M. Mori (Universal Academy Press, Inc.)m 235, 383, 503120 (2003).

[9] V. G. Sinitsyna and V. Yu. Sinitsyna, Astron. Lett. 37(9), 621 (2011)
[10] T.-P. Li and Y.-Q. Ma, Astrophys. J. 272, 317 (1983)

[11] M. Stickel, J. W. Frie, H. Kuehr, Astron. Astrophys. S 80, 103 (1989)

[12] E. Idesawa et al., Publ. Astron. Soc. Jpn. 49, 631 (1997)

[13] N. Isobe et al., Publ. Astron. Soc. Jpn. 53, 79 (2001)

[14] H. Seta et al., Publ. Astron. Soc. Jpn. 61, 1011 (2009)

[15] C. E. Tateyama et al., Astrophys. J. 520, 27 (1999)

[16] E. Valtaoja et al., Astrophys. J. 531, 744 (2000)

[17] D. C. Gabuzda, J. L. Gó mez, Mon. Not. R. Astron. Soc. 320, L49 (2001)

[18] C. E. Tateyama, K. A. Kingham, Astrophys. J. 608, 149 (2004)

[19] G. Ghisellini, et al., Mon. Not. R. Astron. Soc. 301, 451 (1998)

[20] R. C. Hartman et al., ApJS 123, 79 (1999)

[21] A. A. Abdo et al., Astrophys. J. 700, 597 (2009); Astrophys. J. 715, 429 (2010)

[22] M. Hayashida et al., Proc. of 31st ICRC, Lodz (2009)

[23] F. Krennrich, T.C. Weekes et al., Univ. Acad. Press, Inc. 157 (2003)

[24] M. Catanese and T. C. Weekes, Preprint Series No4811, (1999).

[25] A. D. Kerrick et al., ApJ 438, L59 (1995).

[26] J. H. Buckley, Astropart. Phys. 11, 119 (1999).

[27] R. Mukherjee et al., Astrophys. J. 490, 116 (1997).

[28] A. A. Abdo, et al., ApJ 699, 817 (2009).

[29] P. L. Nolan et al., ApJS 199, 31 (2012)

[30] R. C. Gilmore et al., ArXiv:1104.0671

[31] T. M. Kneiske et al., Astron.\&Astrophys. 386, 1 (2002)

[32] T. M. Kneiske et al., Astron.\&Astrophys. 413, 807 (2004)

[33] F. W. Stecker, M. A. Malkan, S. T. Scully, Astrophys. J. 648, 774 (2006)

[34] M. R. Orr, F. Krennrich, E. Dwek, Astrophys. Space Sci. 733, 77 (2011) 